\title{
F-molding: a new production method for largely aspherical mirrors of cordierite.
}

\author{
Kyocera International Inc.: Shinobu Nagata (shinobu.nagata@kyocera.com), Kyocera Corporation: Hidehito Himeno, Yuji Ikeda, Shinji Mukai, Hidenori \\ Yamanokuchi, Takato Honjou,Yuuki Nishi, Shinichi Yamaguchi, Kenichi Hamamura, Junpei Yamaguchi and Masatsugu Kamiura
}

Cordierite is a rigid low thermal expansion coefficient ceramic equivalent to that of low thermal expansion glass but with voids due to forming with raw material powder. To overcome this problem, we propose Flexible Molding (F-molding) as our new fabrication method. With F-molding, the raw material powder is converted to liquid slurry, which is poured into a resin mold, solidified, and directly formed into near net design of a mirror. The mold prepared from F-molding is an effective tool for processing multiple volumes of identical structures such as those used in segmented mirror designs. We introduce details of our fabrication method in F-molding mirror and report its measurement results of optical performances with a $270 \mathrm{~mm}$ diameter sample.

\section{Introduction}

Cordierite ceramic is a promising material with advantages such as longterm dimensional stability $(\sim 3.8 \mathrm{~nm} / \mathrm{m} /$ year $)$, high specific rigidity $(\sim 56.9 \mathrm{GPa} /(\mathrm{g} / \mathrm{cm} 3))[1]$, and high thermal conductivity $(\sim 4 \mathrm{~W} / \mathrm{mK})$ allowing for efficient cooling after thermal processing relative to low thermal expansion glass materials. Soft molded green body of the ceramic prior to sintering allows for machining of its structure without the use of grinding for efficient and cost-effective processing. Cordierite demonstrated its success through development of lithography stage and has shown to achieve $7 \mathrm{x}$ performance improvement as a lens barrel with Subaru Telescope [2].
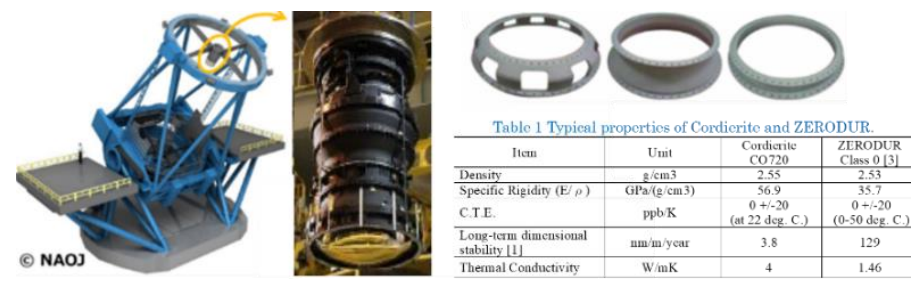

Figure 1 Subaru Telescope (left), Lens barrel (center) and made of Cordierite by KYOCERA (right top). [2]

https://www.youtube.com/watch?v=|7YhIKK1jSM\&feature=youtu.be

However, as a mirror, Cordierite is prone to voids which often appear on polished surfaces generated by destruction of small grains leading to tetrahedral gaps from sphere packing of raw powder material during CIP (Cold Isostatic Pressing) process.

With F-molding, raw powder material is converted to a liquid slurry which is poured into a resin mold, solidifies without sphere packing, and directly forms to near net design of a mirror while potentially reducing void defects. Mold prepared with F-molding also improves productivity of mirrors with a same design and is an advantage in segmented mirrors of extremely large telescopes, and constellation optics.

\section{Flexible-Molding}

In F-molding, a liquid slurry is casted onto a mold prepared from metallic or resin-based model of a final design not limited by its materials. Forming to the final design allows F-molding to bypass green machining process, and the mold is kept for preparation of additional parts towards volume production.

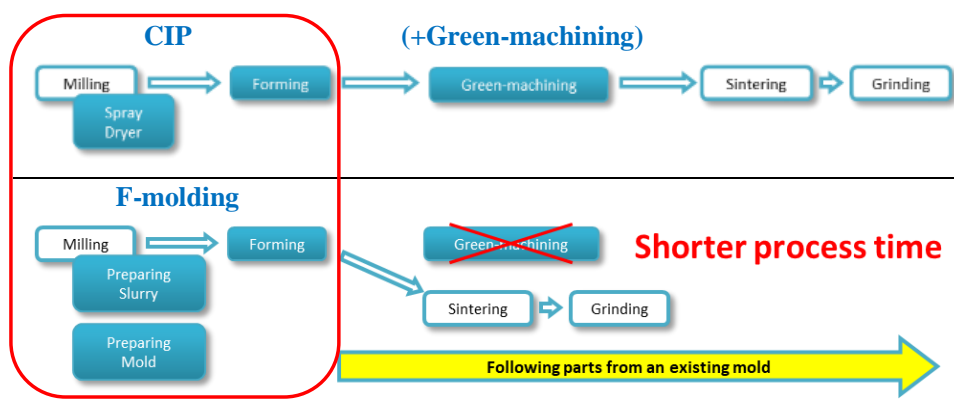

Figure 2: CIP(Top) and F-molding (Bottom) ceramic process flow.

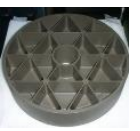

Formed

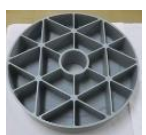

Sintered

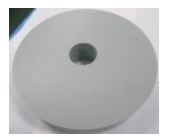

Post grinding

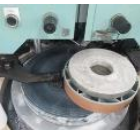

Polishing

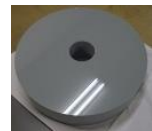

Post polishing
Figure 3: $\varphi 270 \mathrm{~mm}$ F-molding ceramic at each process stage.

Other advantages in F-molding is observed in its liquid slurry-based forming method without a cause for sphere packing and has a potential to reduce voids within the ceramic without the use of HIP treatment.

\section{Conclusion}

F-molding proves to be a process that are efficient at volume production, while being able to maintain comparable material characteristics to HIP treated Cordierite. Below are results obtained through F-molded $\phi 270 \mathrm{~mm}$ mirror sample.

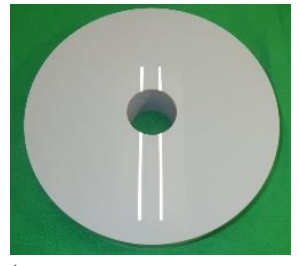

A) $\quad$ 270mm F-molded sample

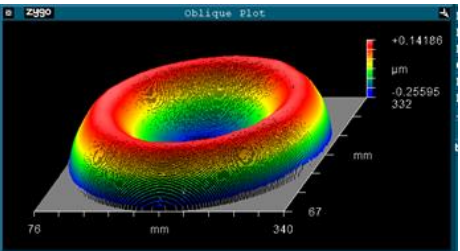

C) Flatness measurement

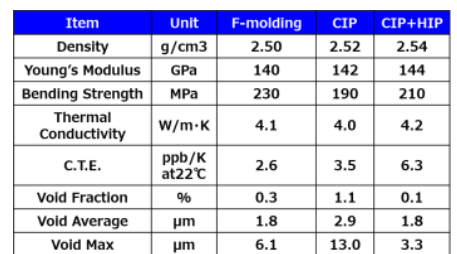

B) Measurement results

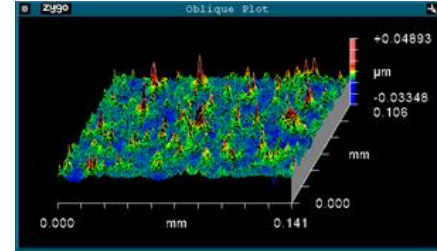

D) Surface roughness
Figure 4: Results from $\varphi 270 \mathrm{~mm}$ F-molded sample evaluation.

1. Flatness $0.39 \mu \mathrm{m} /$ Surface roughness $1.363 \mathrm{~nm}$ achieved.

2. Void fraction $0.3 \%$ /Average void diameter $1.8 \mu \mathrm{m} / \mathrm{Max}$ void diameter $6.1 \mu \mathrm{m}$ achieved.

3. Verified no significant difference in material characteristics of Fmolding to CIP.

4. Achieved greater than $50 \%$ reduction in raw materials utilization relative to CIP forming.

5. Unnecessary machining process would allow for a much more complex structure in its final design.

Processing of $>\varphi 1 \mathrm{~m}$ scale mirrors, 1-month machining time of CIP forming would have significant impact in lead time when processing multiple mirrors of the same size in segmented mirror designs. F-molding on the other hand would only require machining time on a single base model as opposed to CIP forming, and is efficient in processing of segmented mirrors as well as volume parts which may be used in constellation optics.

\footnotetext{
${ }^{1}$ Legero, T., "Interferometric measurements of low thermal expansion materials," FV-No. -43029

2 Yamanokuchi, H., Hagi, S., "Large Ceramics Parts Enhanced Performance of Subaru Telescope by Seven Times" CERAMICS JAPAN, Vol.48 (No. 8), 606-609 (2013)
}

SCHOTT Technical Information, TIE-43: Properties of ZERODUR®
https://www.us.schott.com/d/advanced_optics/e567e0bf-66d1-4757-965e-
a7ca5aa896b7/1.0/schott_tie-43-properties-of-zerodur_us.pdf 